\title{
The influence of inulin on the structural and mechanical properties of dough for the gluten- free biscuit
}

\author{
Irina Krasina ${ }^{1, *}$, Anastasia Strelkova ${ }^{1}$, Nargiz Duishenbek $^{2}$, and Elizabeth Filippova ${ }^{1}$ \\ ${ }^{1}$ Kuban State Technological University, Russia \\ ${ }^{2}$ Kyrgyz State Technical University named after Razzakov, Kyrgyzstan
}

\begin{abstract}
For the production of the low-caloric gluten-free cookies, the fat content was reduced by 50,60 and $70 \%$ in the cookie formula. As the fat level decreased, the hardness of the gluten-free dough increased, and the ejection time of the gluten-free dough rose. The texture analysis showed an increase of the dough hardness. The fat replacing with an equal amount of inulin to some extent reduced the consistency of the dough and its hardness, and its cohesive, elastic and adhesive properties increased. The effect of fat reduction negatively affected the texture of gluten-free cookies. The research showed that the texture of gluten-free cookies is highly dependent on the texture of the gluten-free dough. In addition to the fact that fat is responsible for the dough softness and the cookies crisp texture, it also gives an aroma, taste, mouthfeel and general impression to the finished product. The texture of the cookies improved significantly when the fat was replaced by inulin. Accordingly, the quality indexes of the gluten-free cookies could be significantly improved by the fat replacing with inulin.
\end{abstract}

The growing interest in functional products has prompted to research new ingredients and new ways to incorporate them into the daily diet. In many aspects, food is considered not only from a nutritional point of view, but also as a preventive treatment or even as a various health diseases therapy [1]. Thus, the gluten-free foods can be seen as the functional food for those people who suffer from gluten intolerance or celiac disease, known as gluten-sensitive enteropathy. In addition to the celiac disease (1\%) and the gluten intolerance (6\%), 22\% of the world population avoid eating gluten-containing foods for various reasons [2]. Among the huge mass of consumers the choice preferences of these products largely depend on their food habits and health care. Despite the growing popularity of gluten-free products and the choice offered in supermarkets, the quality of such products leaves much to be desired. They are mainly based on the starchy flour, such as rice or corn, they contain carbohydrates and fats, but they are poor in proteins and other essential nutrients, such as vitamins and minerals, as well as dietary fibers [3,4]. In addition, the texture of such products leaves room for improvement. The absence of gluten, a structural protein, leads to the appearance of a sandy taste in the mouth and the crumply texture, as well as a lack of volume, and sometimes shape. In contrast, cookies do not need a developed gluten network and may therefore serve as a

* Corresponding author: krasina@kubstu.ru 
suitable matrix for the incorporating functional ingredients such as proteins and dietary fibers into the gluten-free food matrices. In addition, they have a relatively long shelf life expiration, good food qualities and are also widely demanded by consumers. The acceptability of these products is usually determined by their taste, aroma and appearance, in which sugar and fat are of primary importance. Fats and sugars give the cookies, including gluten-free, good sensory properties, that is, appearance, texture, taste and mouth sensation, but it is practically not consumed by health-conscious people, because today the large amount of fat consumption becomes one of the main nutritional problems [5,6]. For this reason, despite the important role that fat plays, the researches for the fat content reduction in food products and its replacement with various fat substitutes continue. Fat substitutes are either lipid-like substances or mimetics (protein or carbohydrate substances). The largest group of mimetics are carbohydrate-based ingredients that act imitating the functional and sensory properties of real fats in food systems [7]. In addition to the ability to imitate the fat properties, some mimetics contain functional components, for example, dietary fibers, and therefore can be used to improve the functional product profile [8].

Maltodextrin and polydextrose are the two most popular substitutes for carbohydratebased fats [9]. Carbohydrate-based fat substitutes form a gel- like matrix in the presence of a significant amount of water, resulting in viscous and fluid properties similar to those of fats. However, in the literature there is insufficient information on the fat use as a mimetic in gluten-free inulin cookies. Inulin is a hydrophilic fructose polymer and can therefore act as a carbohydrate fat substitute that acts as a dietary fiber, filler and prebiotic, so the nutritional inulin is an excellent alternative to fat [10]. It has good fat-imitating properties in the combination with water due to the gel forming ability.

The purpose of our work was to study the effect of fat replacement with inulin on the rheology of gluten-free dough and the quality of cookies.

The dough for gluten-free cookies was prepared on the basis of the flour mixture of corn starch, corn flour and buckwheat flour with the addition of caster sugar, eggs and fat. For the preparation of the gluten-free cookie test samples, the fat content was reduced by 50, 60, 70 $\%$ in the formula and was $20 \%$ by weight of all the recipe components in the control sample, and 10, 8 and 6\%, respectively, in the test samples. As a fat mimetic, inulin was added to the formula in such an amount that the total fat and inulin content was $20 \%$ of the weight of the recipe components, i.e. 10, 12 and 14\%, respectively. The textural properties of the glutenfree dough were determined on the Instron 4301 texture analyzer. The biscuits were evaluated by diameter and thickness and their ratio, the tensile strength of the biscuits was measured in a TA-HDi texture analyzer, Stable Micro Systems, UK. The sensory assessment of the biscuits quality was also carried out.

Table 1 shows the effect of the fat impoverishment (F) and the inulin addition (I) on the properties of the gluten-free cookie dough. The consistency measurement method showed the gluten-free dough became harder when the fat content decreased in the formula. However, it should be noted that the higher proportion of inulin does not lead to a significant change in the dough hardness values, which suggests that no further change in the dough consistency occurs above a certain inulin level.

Table 1. The effect of fat and inulin content on the gluten-free dough consistency

\begin{tabular}{|l|c|c|c|c|c|}
\hline \multirow{2}{*}{ Fat content,\% } & \multirow{2}{*}{ Ejection time, sec } & \multicolumn{4}{|c|}{ Texture } \\
\cline { 3 - 6 } & & hardness, $\mathrm{c}$ & cohesion & adhesion, $\mathrm{N}$ & hardness, $\mathrm{N}$ \\
\hline $20 \mathrm{~F}$ & 43 & 20,8 & 0,39 & 0,34 & 0,6 \\
\hline $10 \mathrm{~F}+\mathrm{I}$ & 52 & 26,6 & 0,49 & 0,46 & 0,74 \\
\hline $8 \mathrm{~F}+\mathrm{I}$ & 55 & 29,4 & 0,43 & 0,55 & 0,96 \\
\hline $6 \mathrm{~F}+\mathrm{I}$ & 58 & 34,1 & 0,48 & 0,63 & 1,48 \\
\hline
\end{tabular}


The consistency measurement method showed that the gluten-free dough became harder when the fat content decreased in the formula, however, it should be noted that higher doses of inulin do not lead to a significant change in the dough hardness values, which says that no further change in the dough consistency occurs above a certain level of inulin. The time taken to the dough ejection is directly related to its consistency. The control sample of the glutenfree dough containing a normal fat level (20\%) required 43 seconds for the ejection, while the ejection time for the dough sample with fat containing $50 \%$ less was increased to 52 seconds. The ejection time increased even more to 58 seconds with the fat impoverishment $70 \%$ less. The result shows that the gluten-free dough loses its elastic properties, so it takes more time to press it off.

The texture measurement of the gluten-free dough on the texture analyzer shows that the hardness value increases with a fat content decrease in the cookie formula, while the inulin content increase reduces the hardness degree. The fat replacement with inulin also increased the cohesion properties of the gluten-free dough, although with the inulin dosage increasing, the cohesion of the dough decreased, but in any case the values were higher than in the control sample. The observed increasing of the cohesive properties of the dough can be explained by the lack of a sufficient level of fat in the cookie formula. The cohesive matrix of the dough is due to the development of viscoelastic protein fibrils and is undesirable in the cookie dough, as it leads to the strength increase of the finished product. The results also shows that the resilience or the elasticity of the gluten-free dough is increased by the fat content reducing in the formula and by the replacing it with inulin.

The cookie physical properties changes with the fat content reducing in the gluten-free cookie formula and its replacement with inulin are shown in Table 2.

Table 2. The effect of fat and inulin on the physical indexes of the gluten-free cookies

\begin{tabular}{|c|c|c|c|c|}
\hline Sample & Diameter, $\mathrm{mm}$ & Thickness, $\mathrm{mm}$ & Slackness & Strength, kgS \\
\hline $20 \mathrm{~F}$ & 55,5 & 6,3 & 8,8 & 1,03 \\
\hline $10 \mathrm{~F}+10 \mathrm{I}$ & 56,1 & 6,8 & 8,3 & 1,48 \\
\hline $8 \mathrm{~F}+12 \mathrm{I}$ & 55,5 & 6,8 & 8,2 & 1,72 \\
\hline $6 \mathrm{~F}+14 \mathrm{I}$ & 55,5 & 7,5 & 7,4 & 2,05 \\
\hline
\end{tabular}

The slackness of the cookie was significantly reduced when the fat content was decreased in the formula. The control cookie sample containing a normal fat level had a post-baking diameter of $55.5 \mathrm{~mm}$, which decreased to $50.5 \mathrm{~mm}$ when $70 \%$ of the fat was replaced by inulin in the formula. Similarly, there was a significant increase in the thickness of these backed cookies from $6.3 \mathrm{~mm}$ in the control sample to $6.9 \mathrm{~mm}$ in the sample with the $70 \%$ replacement. Our observations showed that the elastic properties of the gluten-free cookie dough have increased in the absence of fat significant volume. This dough elasticity affects the decrease in the dough slackness and the thickness of the corresponding cookies. The results also showed that the fat level reducing increases the cookies tenacity (Figure 1). 


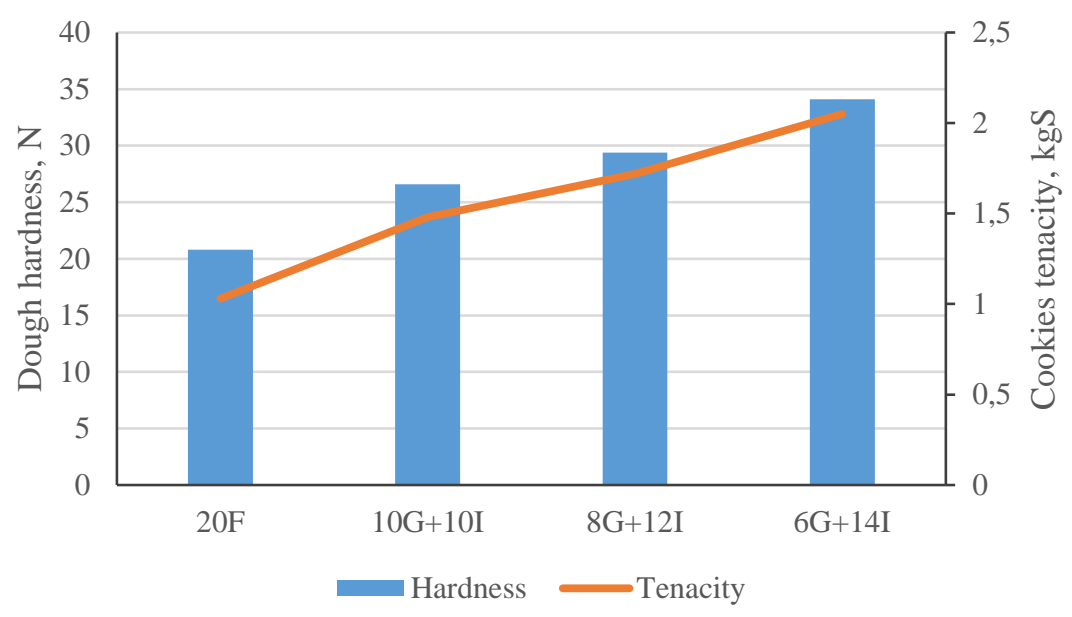

Fig. 1. The inulin effect on the gluten-free dough hardness and the cookies tenacity.

The sensory evaluation of the inulin-containing cookie in comparison with the control cookie sample is shown in Table 3.

Table 3. The sensory evaluation of the inulin-containing cookies

\begin{tabular}{|c|c|c|c|c|c|c|}
\hline Sample & Color & Surface & Texture & Taste & Aroma & $\begin{array}{c}\text { General } \\
\text { impression }\end{array}$ \\
\hline $20 \mathrm{~F}$ & 7,38 & 7,72 & 7,79 & 7,75 & 7,80 & 7,63 \\
\hline $10 \mathrm{~F}+10 \mathrm{I}$ & 7,34 & 7,50 & 7,68 & 7,80 & 7,88 & 7,78 \\
\hline $8 \mathrm{~F}+12 \mathrm{I}$ & 7,42 & 6,89 & 7,37 & 7,19 & 7,63 & 7,69 \\
\hline $6 \mathrm{~F}+14 \mathrm{I}$ & 6,89 & 6,75 & 6,98 & 6,94 & 7,05 & 7,18 \\
\hline
\end{tabular}

The results showed that the color and physical configuration of the cookies improved when the fat was replaced by inulin by $50 \%$. While replacing $70 \%$ of fat, no significant improvement was observed. Although the significant reduction of the cookies tenacity was observed on the texture analyzer, the subjective analysis showed that there was no significant deterioration of the texture of the inulin-containing cookies. A slight improvement in the taste and aroma of cookies with the 50\% fat-to-inulin replacement was noted during the sensory evaluation. The obtained results showed while the fat replacement with inulin the functional and technological properties of the fat-inulin mixture did not fully imitate such pure fat properties as the aroma or taste imparted by the fat in the gluten-free cookie dough formula.

Thus, it can be concluded that by the fat reducing in the gluten-free formula, the dough became harder and its cohesive, elastic and adhesive properties increased. Our research showed that the properties of the gluten-free dough could be improved by the replacing of fat with inulin. The study shows that the texture of gluten-free cookies is highly dependent on the texture of the gluten-free dough. In addition to the fact that the fat is responsible for the dough softness and the crisp texture of the cookies, it also gives the finished product aroma, taste, mouthfeel and general impression. The present study showed that, despite a significant improvement of the texture of the gluten-free dough and cookies, while replacing fat with inulin, the attempt to achieve other functional properties of fat in the gluten-free cookie formula from the soft dough proved to be challenging. 


\section{References}

1. K.M. Murtazina, D.D. Shamova., E.O. Samitov Eurasian Scientific Association. 7-2 (53), 100-102 (2019)

2. I.A. Bavykina, M.V. Pertseva, D.V. Bavykin Medicine: theory and practice. 1, 17 - 18 (2018)

3. N.K. Danovich, I.B. Krasina, O.I. Kazmina, The Proceedings of Higher Educational institutions. Food technology. 1(343), 49-52 (2015)

4. J. Xu, Y. Zhang, W. Wang, Y. Li Trends in Food Science \& Technology, 103, 200 (2020)

5. S.Y.Misteneva, N.A. Shcherbakova, T.V. Savenkova, I.I. Mizinchikova. Food service Industry. 12, 41-47. (2020)

6. T.A. Karachanskaya, I.B. Krasina, N.K. Danovich Scientific-theoretical objectives of the production of the flour confectionery products with the special purpose. (Krasnodar, 2013)

7. M. E. Moriano, C.Cappa, C. Alamprese Journal of Cereal Science 81, 171-178(2018)

8. I.B. Krasina, N.F. Teslenko, N.A. Tarasenko, Y.G. Denisenko, P.S. Krasin University Proceedings. Food technology. 2-3, 62-63 (2011)

9. C. Villemejane, P. Roussel, S. Berland, P. Aymard, C. Michon Journal of Cereal Science, 57 (3), 551-559 (2013)

10. D. Meyer, S Bayarri, A.Tarrega, \& E. Costell Food Hydrocolloids 25, 1881-1890 (2011) 